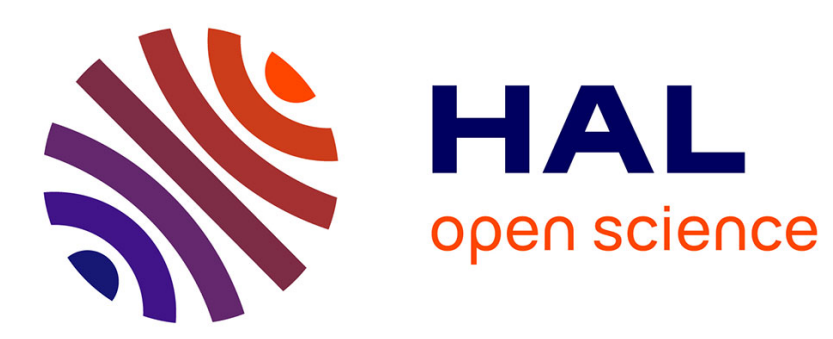

\title{
Interactions médiatisées sur scène
}

\author{
Alain Bonardi, Francis Rousseaux
}

\section{To cite this version:}

Alain Bonardi, Francis Rousseaux. Interactions médiatisées sur scène. Revue des Sciences et Technologies de l'Information - Série TSI: Technique et Science Informatiques, 2010, 29 (8), pp.871-898. hal-01106567

\section{HAL Id: hal-01106567 \\ https://hal.science/hal-01106567}

Submitted on 20 Jan 2015

HAL is a multi-disciplinary open access archive for the deposit and dissemination of scientific research documents, whether they are published or not. The documents may come from teaching and research institutions in France or abroad, or from public or private research centers.
L'archive ouverte pluridisciplinaire HAL, est destinée au dépôt et à la diffusion de documents scientifiques de niveau recherche, publiés ou non, émanant des établissements d'enseignement et de recherche français ou étrangers, des laboratoires publics ou privés. 


\title{
Interactions médiatisées sur scène
}

\author{
Alain Bonardi \\ Ircam, Place Stravinsky, 75004 Paris \\ Université Paris 8, 2 rue de la Liberté, 93526 Saint-Denis \\ alain.bonardi@ircam.fr
}

Francis Rousseaux

\author{
Université Reims Champagne Ardenne, Laboratoire CRéSTIC \\ Ircam, Place Stravinsky, 75004 Paris \\ francis.rousseaux@univ-reims.fr
}

RÉSUMÉ. Prenant acte de la crise des représentations liées à la "graphosphère » et de la montée en puissance des représentations multimédia, constatant l'émergence de l'informatique temps réel dans les arts de la scène, nous avons mené notre recherche depuis plusieurs années sur le thème de la médiation des technologies de l'information et de la communication dans l'écriture des spectacles vivants (théâtre et opéra, principalement), et leur recréation par les spectateurs. Notre perspective est la conception d'environnements informatiques pour la création artistique scénique et sa réception, destinés à trois communautés d'utilisateurs : les créateurs d'œuvres, les performeurs ${ }^{1}$ sur scène, et le public au sens large.

ABSTRACT. Noting that representations belonging to the " graphic sphere » undergo a severe crisis whereas multimedia representations have become more and more powerful and widespread, noticing that realtime computing is emerging in the field of scenic arts, we have carried out our research for several years on the topic of the mediation of information and communication technologies for the design of live shows (theatre and opera, mainly), and their recreation by spectators. Our perspective is the design of computerized environments for artistic creation on stage and its reception, intended for three communities of users: work creators, performers on stage and the audience in a broad meaning.

MOTS-CLÉS. Médiations technologiques sur scène, interactions, environnements virtuels informés, collections figurales.

KEYWORDS. Technological mediations on stage, interactions, virtual informed environments, figural collections.

\footnotetext{
${ }^{1}$ Le performeur désigne tout artiste en situation de jeu scénique. Le terme, issu du performer anglais, incarne la dimension « performative » fondatrice du travail artistique engagé, dépassant l'interprétation de textes, et pouvant aller jusqu'à des formes d'improvisation. Par cohérence, nous conservons le terme anglais performance qui désigne ce travail scénique.
} 


\section{Contexte général}

\subsection{Les mutations de nos représentations}

Notre époque traverse une crise de la représentation au sens large, le plus souvent relevée dans le domaine politique, mais évidemment présente dans l'art. Elle s'éprouve aussi bien au Centre Beaubourg qu'au Parlement. La forme actuelle de la crise de représentation «peut s'interpréter comme un retour du réel, aux multiples manifestations. Le réel se rappelle quand l'urgence du présent vient supplanter la représentation. [...] Du côté des artistes, on assiste à une coupure grandissante entre les arts qui relèvent du récit, ou d'un pôle littéraire de référence, et ceux qui produisent des ambiances et des rythmes [...] Patience dans le récit, impatiences médiatiques...» [Bougnoux 2006].

A l'opposé des arts liés au texte, qui perdent une partie de leur autorité, le multimédia interactif apparaît comme un terrain idéal pour cette quête de présentisme et de manifestations presque vitales, surtout le monde des jeux vidéo, qui propose l'immédiateté, les raccourcis de réflexion, les mises en présence instantanées qui répondront à ces désirs tout en les façonnant.

Ces évolutions et oppositions de représentations se manifestent de bien des manières, mais retenons-en deux qui nous paraissent particulièrement symptomatiques :

Le statut récemment accordé au geste dans la mise en scène et la création dramaturgique non dansée, appartenant pourtant à la sphère de l'écrit. De simple illustration d'une rhétorique dans l'approche classique, le geste devient chez certains metteurs en scène comme Bob Wilson le vecteur d'une expression cohérente, et presque autonome. Son importance nouvellement acquise, voire son indépendance, manifestent un approfondissement d'approches non exclusivement textuelles de l'opéra. Les incursions de chorégraphes dans la mise en scène d'opéra, comme Pina Bausch pour Le Château de Barbe-Bleue de Bartok (Festival d'Aix-en-Provence, 1998) introduisent une autonomie et une liberté du geste par rapport à une partition et un livret.

L'irruption des écrans sur scène, qui se manifestent dans toute la sphère du spectacle scénique et dans toutes les cultures [Peyret 1998], [Zeppenfeld-Rosaz \& Le Brian-Prada 1998].

\subsection{La calculabilité du spectacle et les transformations de l'altérité induites par les dispositifs numériques sur scène}

Evoquer l'ample question de la médiation des interactions sur scène peut se faire de multiples façons. L'ouvrage de référence de Steve Dixon [Dixon 2007] élabore une vaste et riche perspective historique et thématique, s'intéressant à toutes les formes spectaculaires 
faisant appel aux «new media $»^{2}$ au sens le plus large. Quant au volume dirigé par Béatrice Picon-Vallin [Picon-Vallin 1998], il explore au contraire en profondeur un point précis, celui des nouvelles manifestations des écrans sur les scènes de théâtre. Les musiciens comme Philippe Manoury [Manoury 2007], précurseur du temps réel dans les œuvres mixtes associant instruments acoustiques et ordinateurs, y compris à l'opéra, proposent de leur côté une lecture selon deux axes orthogonaux : le paradigme de la partition, versus le paradigme de l'instrument; les deux sont aisément extensibles à tous les arts de la scène.

A notre connaissance, il n'existe aucune approche systématique du domaine selon les catégories de l'informatique, non pas en classant les productions scéniques par technique implémentée, mais selon les grandes questions qui traversent cette science. Certes, le champ d'étude serait réduit aux cuvres faisant appel à des programmes informatiques, ce qui représente actuellement l'énorme majorité des créations, et ferait l'impasse sur un certain nombre d'œuvres plus anciennes faisant appel à des dispositifs hardware. Depuis le début des années 2000, les deux auteurs abordent le champ créatif de la performance numérique en le plongeant dans des problématiques informatiques ([Rousseaux 2006b], [Bonardi 2008]), tout en validant les résultats de leurs travaux lors de la production de spectacles professionnels. Ces derniers ne sont pas de simples faire-valoir de laboratoire : ce sont d'authentiques spectacles poussés par des artistes se posant la question de l'artefacture non pour les effets que l'on peut en attendre, mais pour les questions que l'on peut en tirer.

Dans cette perspective, un cadre d'analyse du domaine des interactions médiatisées sur scène pourrait être la dyade proposée par Alan Turing [Turing 1995] : la machine de Turing versus le test de Turing ; d'un côté la calculabilité de la scène ; de l'autre, le dialogue homme-machine. Nous en donnons quelques exemples :

- Sur le premier versant, nous pouvons partir du compositeur Richard Wagner (18131883) auquel est souvent associé le concept « d'œuvre d'art totale » [Bablet 1995]. Mais le plus intéressant dans notre perspective est l'approche nouvelle proposée par le musicien, qui considère les différents aspects de la scène comme paramétrables et donc potentiellement calculables (notamment l'acoustique, comme en témoigne le théâtre de Bayreuth). Le mouvement de fonctionnalisation de la scène était lancé, qui aboutirait dans les années 1980- 1990 à des plateformes informatiques de traitement temps réel comme Max/MSP ou PureData, désormais capables de piloter sons, lumières, images, vidéos, etc. et d'intégrer toutes sortes de développements logiciels allant du traitement du signal à l'informatique symbolique.

- Sur l'autre versant, celui du dialogue homme-machine, on ne compte plus les productions mettant en situation soit les performeurs soit les spectateurs face à des machines au sens large. Ainsi, dans Blue Bloodshot Flowers de Susan Broadhurst

\footnotetext{
${ }^{2}$ Dixon fait toutefois l'impasse sur la performance musicale (la musique est pourtant à l'origine des plateformes temps réel utilisées dans l'ensemble des arts de la scène) et ignore une bonne partie de la production scénique non anglophone.
} 
(2001), un avatar intelligent appelé Jeremiah apparaît à l'écran sous la forme d'un visage capable de suivre des yeux la performeuse Elodie Berland, et de manifester une expression de colère, de tristesse ou d'amour. Depuis 2008, l'artiste multimédia Agnès de Cayeux explore l'introspection et la confrontation du spectateur à des avatars Internet cristallisant leurs désirs (exemple de l'œuvre Alissa1969 Seriman).

\subsection{Thématiques de recherche et méthodologie}

Nous prenons bien entendu acte de la crise de la représentation relative à la " graphosphère » et de l'irruption massive des représentations multimédia; nous insérons toutefois notre recherche dans un cadre qui n'abandonne pas pour autant les textes au sens large au profit de la performance, mais joue sur plusieurs formes de représentation. Notre terrain de pratique sera essentiellement le théâtre et l'opéra, allant des formes les plus écrites aux plus proches de l'improvisation. D'une manière générale, nous nous intéressons aux formes ouvertes, c'est-à-dire allant de structures temporelles combinatoires à choix multiples ${ }^{3}$ se réalisant en situation scénique à des canevas complètement écrits, dans lesquels l'informatique contribue à « ouvrir » l'espace de l'interprétation.

Nous prenons également en compte l'émergence de l'informatique temps réel dans les arts de la scène et de ses communautés de développeurs/utilisateurs; et bien entendu, la convergence numérique de tous les contenus, qui est une condition nécessaire de ces développements.

Dans ce contexte, nous posons notre démarche exploratoire articulant réflexions et réalisations autour d'environnements informatiques pour la création artistique scénique. Comment les questions centrales de l'informatique peuvent-elles intervenir dans les processus de conception et de représentation de spectacles de théâtre et d'opéra ? Et réciproquement, les pratiques scéniques peuvent-elles enrichir les grandes problématiques de l'informatique?

Dans cette approche, navigant entre informatique et monde de la scène, nous avons travaillé sur les thématiques et les spectacles suivants :

- Classification et mise en scène : la mise en scène comme activité de classification, et la mise en scène de la classification. Cette question rejoint celle tout aussi importante de la co-construction homme-machine d'un espace dramatique pendant la performance. L'accent est mis sur la conception d'Environnements Virtuels Informés scéniques associant performeurs, public et machines, tant du point de vue technique que du point de vue cognitif. Ces questions ont été abordées dans la pièce de théâtre La Traversée de la nuit et dans l'opéra numérique en forme ouverte Alma

\footnotetext{
${ }^{3}$ Ce serait l'équivalent sur scène de ce que proposait le cinéaste Alain Resnais en 1993 dans son dyptique Smoking/No smoking : prendre ou ne pas prendre une cigarette et la suite sera un film différent.
} 
Sola.

- Ressaisissement des intentions humaines et mise en scène [Bouchez \& LeleuMerviel 2001] : il s'agit d'étudier des dispositifs informatiques « intelligents » pour la création de scénographies numériques professionnelles, positionnés dans ses processus : l'enjeu est de susciter des interférences productives entre les « traces » au sens large (écriture, geste, musique, vidéo, etc.) des concepteurs humains et des programmes informatiques pertinents, ainsi que de permettre une meilleure autonomie de l'artiste utilisateur. La première étape de ce travail est notre contribution à la pièce de théâtre Les Petites Absences.

Le travail sur ces questions passe par trois aspects indispensables :

- La production d'outils et d'environnements réutilisables, de niveau sémantique dans des environnements orientés originellement vers le traitement du signal, à mettre à la disposition de la communauté des utilisateurs des outils artistiques « temps réel ».

- Le développement de maquettes logicielles intégrées dans les spectacles, utilisant les outils précédents.

- L'expérimentation avec des performeurs avant toute répétition et pendant la préparation du spectacle.

Ajoutons que ces questions centrales de l'informatique s'inscrivent dans nos pratiques sociales et culturelles. Nous ne négligeons pas cet aspect. Constater la montée en puissance du multimédia et des jeux vidéo ne suffit pas. Il s'agit de prendre une distance critique par rapport à ces nouvelles représentations, pour en dégager des paradigmes, les expérimenter, notamment en contexte scénique, et ce non seulement pour en saisir la portée artistique mais également les résonances dans la société. Le présent article se propose de rendre compte de cette recherche, de ses thématiques (classification, ressaisissement des intentions) et de sa méthodologie.

\subsection{L'évaluation de la démarche}

La validation de notre démarche s'avère délicate. Nous sommes d'une certaine manière tendus entre « le Vrai » et « le Beau » : « le Vrai » de nos algorithmes et de nos conceptions, et « le Beau » des spectacles produits grâce à eux. La chose est d'autant moins aisée que nous nous intéressons plus particulièrement, comme nous l'avons dit au paragraphe précédent, aux formes ouvertes, déterministes mais pas déterminées.

Une approche sèche et analytique évaluerait les trois attendus des projets: la réalisation d'outils génériques pour la communauté « temps réel » des programmeurs tournés vers le monde du spectacle; le développement de maquettes logicielles intégrées aux spectacles ; les spectacles produits en eux-mêmes. 
Les deux premiers sont des logiciels auxquels on peut appliquer tout un ensemble de savoirfaire issus de très nombreux travaux, par exemple en termes de robustesse ou de qualité du logiciel. En ce qui concerne les réalisations « temps réel », même si nous ne sommes pas dans un cadre contraint de temps réel critique, nous pouvons nous appuyer sur des travaux de «l'école française » dans ce domaine, par exemple [Boniol \& al. 2003]. Nous pourrions également évaluer comment et à quel point les outils génériques mis en téléchargement libre sont adoptés par la communauté.

Quant à l'évaluation des spectacles produits, elle serait bien entendu à mener par d'autres, et ne pourrait concerner les aspects esthétiques, seulement la rencontre avec les publics ; la question pourrait faire l'objet d'un article entier; nous renvoyons le lecteur vers les travaux dans le domaine de la réception des œuvres [Pistone 1994].

En termes académiques, comment évaluer les apports entre disciplines nés de cette recherche ? Il y a bien entendu les publications communes arts-sciences faites par les auteurs. Il s'agit plus largement de contribuer aux mouvements actuels de rapprochement entre disciplines, notamment à la polarisation constatée de l'informatique vers les industries culturelles, comme le constate François Pachet [Pachet 2004] : «les problèmes nourriciers de l'informatique évoluent avec la société qui les a créés».

\section{Classification interactive et mise en scène : vers des Environnements Virtuels Informés}

\subsection{Echapper à l'omnipotence des ontologies a priori}

Comme le montre Jean-François Perrot [Perrot 1994], la notion d'objet a connu un formidable essor : à la fois dans notre imaginaire collectif avec le développement de la société de consommation tel que l'analyse Jean Baudrillard [Baudrillard 1988], et dans l'aspiration des informaticiens à systématiquement s'orienter vers la programmation par objets. Elle n'est pas sans influence sur la création artistique, si l'on considère le « propopera $»^{4}$ de Peter Greenaway, 100 objets pour représenter le monde.

La mise en scène de théâtre et d'opéra n'échappe pas à la réification. En effet, face à un texte de théâtre, chaque metteur en scène souhaite proposer sa lecture/interprétation, ce qui suppose toujours un effort pour créer une ou plusieurs formes. Dans le cas du théâtre, elle commence classiquement par l'établissement d'une ontologie synthétique de la dramaturgie : on y décrit les personnages sous forme de types (ce en quoi le théâtre de boulevard par exemple excelle avec son trio, mari, femme et amant !), et en les « instanciant » : on indique le nom du personnage et sa situation au début de la pièce.

\footnotetext{
${ }^{4}$ Littéralement, « opéra d'accessoires », joué pour la première fois en 1997 au Festival de Salzbourg, sur une création musicale de Jean-Baptiste Barrière.
} 
Ainsi, dans les Fourberies de Scapin de Molière, Géronte est une instance du type du « vieillard ». L'ontologie d'une dramaturgie peut également être structurée par des situations-types. Personnages et situations ne sont pas pensés comme singuliers mais bien comme des cas particuliers de modèles plus larges [Banu 2005].

Le déroulement de la pièce propose des variations d'instanciation. Dans le théâtre baroque est posée la question de la permanence du personnage comme « caractère »: Molière, dans Le Bourgeois Gentilhomme, s'interroge sur la possibilité de transformer radicalement son Monsieur Jourdain. La question est de savoir si l'ontologie peut résister à d'éventuelles variations d'instanciation extrêmes.

Les chercheurs dans le domaine des fictions interactives génératives ne se sont pas privés de ce type d'ontologies, pour instancier des situations et des narrations, par exemple dans des jeux. Ce type de modélisation de personnages et de situations conditionne de plus en plus l'activité dramaturgique, à mesure que l'écriture fictionnelle se présente sous le visage d'un savoir clé-en-main qui peut s'apprendre.

Ce mouvement est à mettre en parallèle de celui de l'informatique vers les environnements à objets. Pourtant en informatique, il existe une autre approche de la similarité : c'est l'approche typique de la fouille de données interactive, dans laquelle on représente l'exemple comme une spécialisation de l'ensemble des cas, et où l'on cherche d'autres spécialisations voisines, mais sans disposer par avance d'une ontologie. L'utilisateur accepte de façonner ces regroupements à la main avec l'aide interactive de la machine, de manière ad hoc. Il s'agit d'une approche en extension ${ }^{5}$ : façonner une similarité revient à façonner une liste de contenus de forme similaire par des opérations rectificatives successives mobilisant le calcul numérique et l'instanciation (du côté du système informatisé), en interaction interprétative avec des actions rectificatrices sur les contenus et leurs formes (du côté de l'utilisateur, provoqué par les propositions de la machine).

De nombreuses démarches utilisent les nouvelles technologies pour susciter d'autres modalités de mise en scène que celles fondées sur les ontologies des personnages et des situations : par exemple, l'utilisation de la réalité virtuelle telle que la pratique Mark Reaney [Reaney 2001]; ou encore l'utilisation des nouvelles technologies comme décor interactif: dans sa mise en scène de la pièce Orgia de Pasolini créée en 2001 au Granit, Scène Nationale de Belfort, le metteur en scène Jean Lambert-Wild constitue un décor à partir de représentations de cellules projetées sur un écran coupant la scène, sorte de « jeu de la vie » évoluant selon des informations physiologiques captées sur les comédiens. Ces conceptions du théâtre tentent de sortir de celles mises en avant par le Storytelling [Cavazza \& al. 2002]. $\mathrm{Du}$ point de vue informatique, ce dernier s'inscrit dans une approche formelle de la similarité reposant sur les ontologies, plus précisément sur la notion de similarité par les

\footnotetext{
${ }^{5}$ L'extension est l'ensemble des objets auxquels s'applique un concept.
} 
ontologies.

La recherche que nous avons menée en 2002-2003 sur le spectacle La Traversée de la nuit ${ }^{6}$ interroge ces approches assimilées à la fouille de données interactive au niveau de la mise en scène de théâtre.

\subsection{Réalisation : La Traversée de la nuit}

Comment abandonner le système si robuste des ontologies pré-définies et pré-validées par le texte de la pièce, sur lequel sont construites une bonne partie de nos habitudes culturelles ? Une mise en scène peut-elle ne plus se conformer à une ontologie mais tenter d'échapper à la spécification a priori en s'appuyant sur des interactions multimodales accumulées dans le temps ? C'est le sens de la recherche menée ici.

Texte de Geneviève de Gaulle-Anthonioz, évoquant son emprisonnement au cachot du camp de Ravensbrück à la fin de la $2^{\mathrm{e}}$ Guerre Mondiale, La Traversée de la nuit porte le récit d'une mémoire. Pour servir ce propos et ces intentions de mise en scène, nous avons déployé un dispositif de communication redondant et multimodal entre le public, un personnage et un système artificiel complexe, centré sur une circulation intense des émotions. L'idée était de jouer de manière concurrente sur les temporalités spécifiques du sonore et du visuel. Il s'agit de créer un effet de surdétermination et de saturation de l'espace de communication et de liberté, contraignant ainsi les acteurs et les spectateurs à imaginer chaque soir un espace singulier d'espérance et de lumière, en travaillant sur différentes strates de la mémoire hantée de l'auteur.

\footnotetext{
${ }^{6}$ La Traversée de la nuit, texte de Geneviève de Gaulle-Anthonioz, mise en scène : Christine Zeppenfeld, conception multimédia : Alain Bonardi et Nathalie Dazin, création images : Julien Piedpremier, musique : Stéphane Grémaud, comédienne : Valérie Le Louédec, danseuse : Magali Bruneau. Création le 21 novembre 2003 au Centre des Arts d'Enghien-les-Bains.
} 


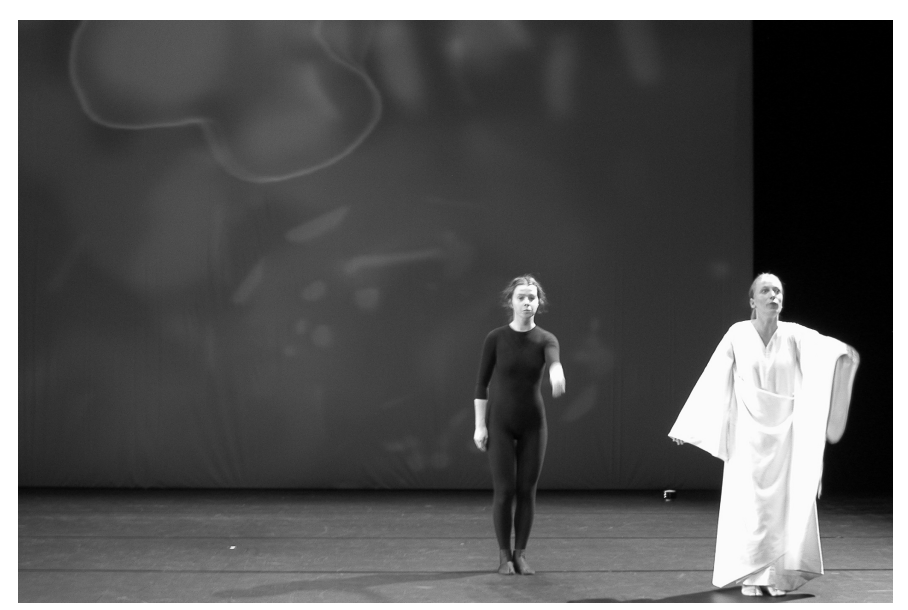

Figure 1. Exemple d'image générée en fond de scène à partir d'états de la voix de la comédienne (en blanc : Valérie Le Louédec), entraînée par la danseuse (en noir : Magali Bruneau). Photographie : Julien Piedpremier.

Précisons les choses en entrant dans la définition du dispositif imaginé par les concepteurs multimédia à partir des options artistiques définies par le metteur en scène (cf. schéma de la figure 2):

- Le système informatique multimédia temps réel mis en œuvre ${ }^{7}$ est constitué en entrée d'un réseau de neurones (perceptron multi-couches) destiné à reconnaître des états émotionnels dans la voix de la comédienne et en sortie d'un système multi-agents générateur d'images projetées sur l'écran. Le réseau de neurones a été entrainé en mode supervisé pendant plusieurs mois par rapport à une liste d'états émotionnels choisis par le metteur en scène, et imposés à la comédienne pendant son travail d'apprentissage.

- La voix en entrée est traitée phrase par phrase, chacune donnant lieu au calcul d'un vecteur de douze composantes pour chacune : quatre d'entre elles concernent la prononciation des voyelles (formants), quatre d'entre elles représentent le caractère bruité de la voix et donc la prononciation des consonnes ; les quatre derniers paramètres s'attachent à la prosodie (agrégateurs sur l'amplitude de la voix). Pour chaque vecteur présenté en entrée, le réseau de neurones fournit un état émotionnel « reconnu ».

- Le système multi-agents permet la génération en temps réel d'images projetées en fond de scène. Les agents sont des ensembles de pixels (nommés « inserts » sur la figure 2) qui s'insèrent dynamiquement dans un film en " toile de fond » imposée. Chaque agent est associé à un ensemble de ressources multimédia, soit des images fixes soit de courtes vidéos. Peuvent varier sa luminosité (de 0 à $100 \%$ ), sa taille à l'écran (étirement ou contraction), sa position, et le dosage des trois couleurs

\footnotetext{
${ }^{7}$ Développé à partir de la plateforme de traitement du signal temps réel Max/MSP/Jitter. Site Internet : http://www.cycling74.com
} 
primaires (rouge, vert, bleu). Dans la version initiale, le système comportait une centaine d'agents. Si nous considérons les caractéristiques usuelles des systèmes multi-agents [FERBER 1995], nous obtenons les niveaux suivants :

- Chaque agent possède un modèle psychologique réduit de sensibilité (positive ou négative), qui réagit selon les séquences de texte, aux états émotionnels manifestés par le réseau de neurones. Il en résulte, en fonction de ce qu'indique le réseau de neurones, et en fonction des poids de sensibilité, une humeur qui conditionne leur « énergie » à accomplir les tâches à mener.

- Les agents coopèrent à un objectif qui est l'optimisation d'une fonction d'utilité de l'image, qui exprime des « buts » scénographiques, par exemple : recouvrir l'écran tous ensemble, disparaître au profit de la trame de fond, etc. La fonction d'utilité est différente pour chaque séquence de texte. Elle est évaluée par un agent-observateur. Un algorithme du gradient à pas fixe est utilisé pour l'optimisation de la fonction d'utilité.

- Les agents se coordonnent dans l'exécution de ce but commun par rapport à l'état émotionnel reconnu par le réseau de neurones, selon un mécanisme de compensation d'humeur : ceux qui sont « d'excellente humeur » (grande valeur positive) concèdent un peu de leur ardeur à ceux qui ont une humeur " très négative $»$.

- Les agents communiquent entre eux, deux à deux, à période fixe, en se transmettant leurs humeurs respectives.

- L'environnement des agents est constitué des états émotionnels reconnus par le réseau de neurones, du repère d'événement indiquant à quel endroit on se trouve dans la pièce, de valeurs propres à la séquence correspondante du texte, et des indications d'un agent- observateur indiquant les qualités de l'image globale construite. 


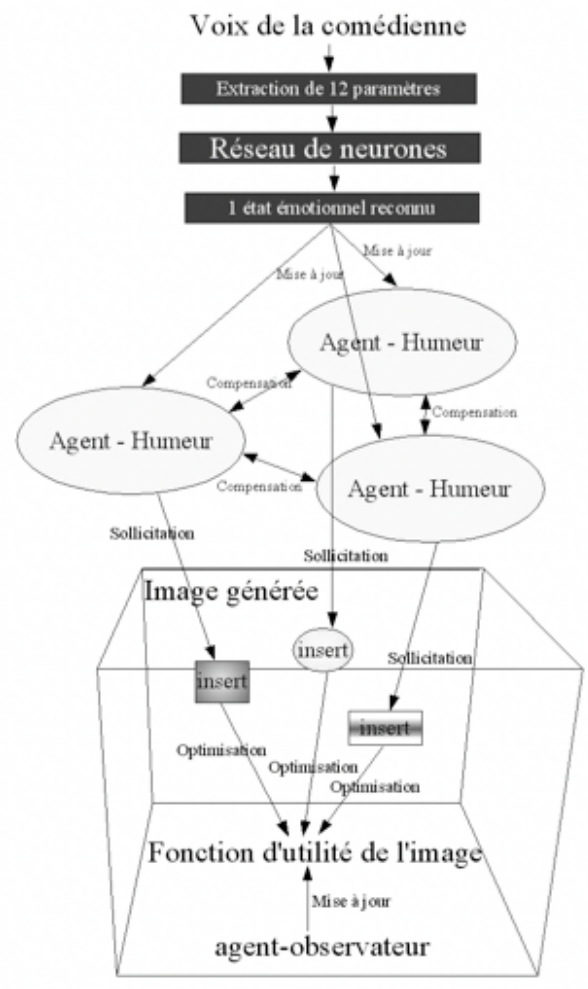

Figure 2. Schéma de fonctionnement de la machinerie interactive de La Traversée de la nuit. Source : Alain Bonardi.

D'une manière qui garde pour les protagonistes sa part de mystère, tout en produisant des rationalités (les acteurs développent des affinités avec le dispositif, notamment lors des phases d'apprentissage), la variation émotionnelle de la voix donne naissance à un avatar visuel. Les interactions primaires entre les spectateurs et le personnage-double s'en trouvent ainsi singulièrement complexifiées et mises en abyme (cf. figure 3), chacun pouvant voir l'émotion qu'il/elle entend par ailleurs, et réagir en conséquence, y compris spatialement pour les acteurs, qui peuvent être tentés de faire jeu avec les mobiles projetés. On conserve alors au niveau dramaturgique le type de stratégies d'écriture citées par Philippe Manoury au niveau musical : "les phénomènes de mémoire, de prémonition, la construction de formes hybrides, les stratégies de préparation et de conclusion, les transitions, les proportions, les courts-circuits » [Manoury 2007]. 


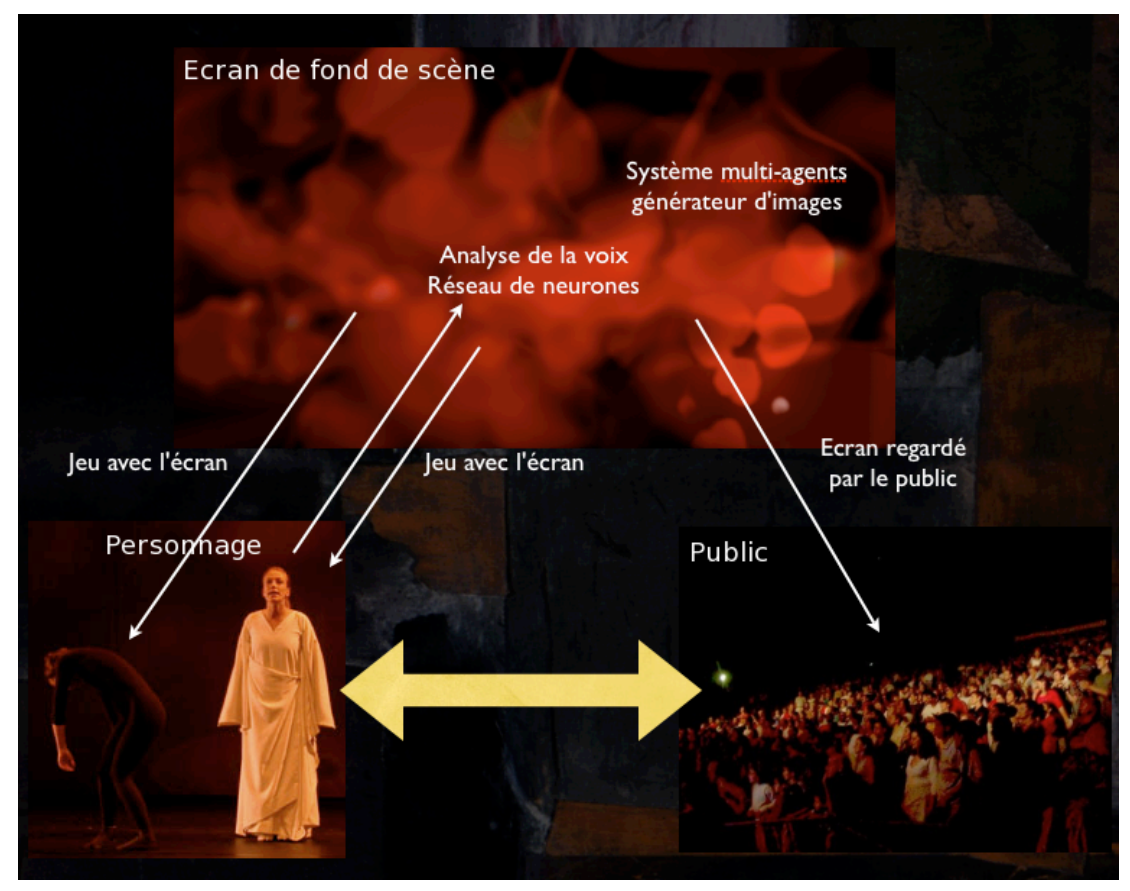

Figure 3. Ensemble des interactions dans La Traversée de la nuit. Source : Alain Bonardi.

\subsection{Vers des Environnements Virtuels Informés Scéniques}

Les mondes virtuels sont habituellement associés aux idées d'immersion ainsi qu'à des conceptions artistiques inspirées par l'univers du jeu vidéo, plus rarement à la création musicale et scénique contemporaine.

Certaines conceptions liées à la réalité virtuelle semblent pertinentes pour rendre compte des situations de jeu musical ou théâtral sur scène en dialogue avec des ordinateurs, dans des environnements scénographiques spatialisés (projections sonores et visuelles multiples). La situation d'interaction qui associe le personnage, incarné par deux comédiennes, le public et le système informatique, nous entraîne sur le versant de la " réalité augmentée ». Nous remarquons que les démarches originales que nous avons évoquées, celles de Reaney, Lambert-Wild, ou les nôtres, ont en commun d'utiliser la réalité virtuelle ou la réalité augmentée comme base d'un dispositif dramaturgique.

Il apparaît que les environnements virtuels sont largement concernés par la dichotomie entre classification en intension et classification en extension. En première approximation, nous avons d'un côté un intérêt pour la modélisation du monde qui s'appuie largement sur des ontologies a priori ; de l'autre, un intérêt pour la modélisation de l'interaction.

Bien que non-immersif, le système homme-machine utilisé dans La Traversée de la nuit relève des Environnements Virtuels Informés, proposant une élaboration progressive de connaissances formant, au fil des interactions, une base de la dramaturgie [Rousseaux \& 


\section{Bonardi 2005].}

Nous souhaitons dans ce travail parvenir à affadir la spécification a priori tout en conservant le texte, en proposant qu'une mise en scène ne soit plus contrôlée et spécifiée par des variations d'instanciation dans des ontologies de personnages et de situations, mais davantage par un glissement de situation contrôlé par la situation elle-même. Le texte de La Traversée de la nuit se prête à une telle approche, et le travail de mise en scène a été conçu non sur l'illustration des situations relatées, mais sur les états mentaux du personnage. L'approche du metteur en scène Christine Zeppenfeld est fondée sur des conditions de possibilité d'une dramaturgie : donner les bases des déplacements, les bases des rythmes de déclamation du texte, mais éviter toute indication de sentiment lié à une narration : au contraire, la consigne est celle d'une grande neutralité, permettant d'ouvrir aux plus infimes variations.

Ce type d'approche nous paraît tout à fait intéressant au théâtre. La validité de la démarche n'en reste pas moins un point méthodologique difficile. Nous notons simplement que le dispositif ainsi construit était robuste, puisqu'il a fonctionné correctement sur scène, et qu'il a « fictionné » auprès de ses utilisateurs pendant plus d'une année : il a été accepté et intégré aussi bien par les comédiennes que par le metteur en scène, et a suscité des initiatives de mise en scène en situation souvent jugées intéressantes. Remarquons qu'il y a eu des soirées d'interactions réussies, et des soirées plus ternes avec des représentations qui manquaient d'énergie : il est impossible de faire la part des choses entre les comédiennes et l'ordinateur... peut-être faudrait-il solliciter nos collègues économistes pour modéliser ces soirées « en repli », autant individuellement que collectivement.

Nous proposons d'aller plus loin dans cette recherche, en rendant explicite cette démarche de classification comme élément moteur de la dramaturgie, alors qu'elle n'est pas figurée en tant que telle dans la production de La Traversée de la nuit. C'est l'objet du projet Alma Sola, fondé sur une forme ouverte assistée par ordinateur.

\subsection{La classification comme moteur d'un opéra numérique en forme ouverte}

Alma Sola ${ }^{8}$ est un opéra numérique sur le thème de Faust, désormais féminin. Le personnage joue contre une machine informatique " surpuissante ", outil de dialogue, de connaissance et de contrôle. Grâce à cet ordinateur méphistophélique, Faust peut créer son propre opéra, en enchaînant librement des blocs pré-composés : il en existe une trentaine,

\footnotetext{
${ }^{8}$ Opéra numérique en forme ouverte pour deux sopranos, guitare et cor (créé en octobre 2005 au Cube, à Issy-lesMoulineaux; dernière reprise: avril 2008 à Vilnius lors d'une résidence conjointe Centre Culturel Français / Conservatoire de Vilnius / Académie des Beaux-Arts de Vilnius) ; composition et création logicielle : Alain Bonardi, livret et mise en scène : Christine Zeppenfeld, direction musicale : Ignazio Terrasi, création images : Julien Piedpremier et Nicolas Hoareau, sopranos : Caroline Chassany et Claire Maupetit, guitare : Gabriel Bianco, cor : Philippe Durand. Nous renvoyons le lecteur au site Internet consacré à cette œuvre : http://www.almasola.net
} 
regroupés en univers thématiques : amour, plaisir, opulence, pouvoir, connaissance. L'opéra cesse lorsque Faust quitte le jeu. Il s'agit d'une forme ouverte dont le modèle dramaturgique est celui du jeu vidéo ou du CD-ROM plutôt que le modèle narratif linéaire de l'opéra traditionnel. Comme pour La Traversée de la nuit, l'idée a été d'élaborer un ensemble interactif, en choisissant des technologies et des méthodes informatiques en adéquation avec les choix faits par les créateurs et le metteur en scène.

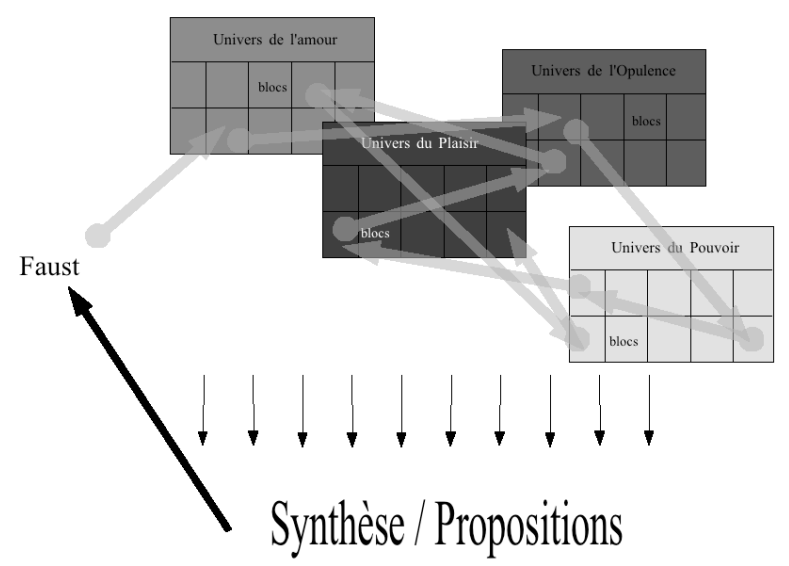

Figure 4. Schéma de fonctionnement de l'opéra Alma Sola. Source : Alain Bonardi.

L'œuvre fonctionne ainsi : Faust choisit un bloc, qui est joué et chanté. Puis l'ordinateur propose une ou plusieurs continuations possibles, c'est-à-dire des blocs qui lui semblent pouvoir être enchaînés avec celui qui vient d'être donné. Enfin, Faust fait son choix qui est signalé aux autres intervenants (musiciens et régie). Dans la version actuelle de l'œuvre, ce choix se fait en manipulant avec une télécommande de jeu vidéo ${ }^{9}$ les fragments d'opéra sur l'écran de fond de scène.

L'enjeu est ici la construction, dans le temps et dans l'espace de la représentation, de la forme de l'œuvre en ordonnant dans l'espace et dans le temps des représentations informatiques de ses fragments.

\subsection{La constitution de collections figurales sur scène}

A ce stade de notre réflexion, les collections se sont imposées à nous pour rendre compte de la nature de l'activité organisatrice et de son caractère compulsif.

A première vue, les collections semblent être plus proches de l'ordre classificatoire que du désordre (qu'il se donne comme tas, amas, cohorte, vrac ou autres fatras) : à tout le moins une collection paraît-elle toujours viser un ordre ou être mise sous la coupe d'un ordre,

\footnotetext{
${ }^{9}$ Modèle choisi : la Wiimote.
} 
même s'il reste provisoirement incomplet et inachevé. Le cabinet de curiosité des savants n'est-il pas exemplaire de la destination des collections, qui est de tomber sous une classification, sous le coup d'une procédure de catégorisation et, finalement, de tri ? Quant aux collections de timbres (pour prendre un autre exemple), n'attendent-elles pas leur complétude catégorielle par l'achèvement des séries commencées

Pourtant, quelque chose résiste à ce rapprochement, et les collections demeurent rebelles à l'idée même de classification. C'est ainsi qu'elles peuvent se trouver repoussées aux « antipodes » des classes, jusqu'à côtoyer les singularités, partageant avec elles leur destin, qui est d'échapper définitivement à toute tentative de rangement.

C'est qu'il faut distinguer ici les collections figurales des collections non-figurales. Cette subtile distinction, introduite dès les années soixante-dix par Piaget et ses équipes [Piaget \& Inhelder 1966] de recherche en psychologie de l'enfant, éclaire en effet la situation d'un jour intéressant : d'une part, il existe certes des collections (non-figurales) qui supportent d'être modélisées par des classes, parce qu'elles sont affranchies de toute intrication avec leur spatialisation (et en cela déjà toutes proches des classes, dont elles n'ont à envier que la complétude formelle); d'autre part, il existe aussi des collections dites figurales parce que leur disposition dans l'espace se fait selon des configurations spatiales qui prescrivent leur signification concurremment aux considérations typiques de la signification des classes [Piaget \& Inhelder 1966] : "En un mot, la collection figurale constituerait une figure en vertu même des liaisons entre ses éléments comme tels, tandis que les collections nonfigurales et les classes seraient indépendantes de toute figure, y compris les cas où elles sont symbolisées par des figures et malgré le fait qu'elles peuvent ainsi donner lieu à des isomorphismes avec des structures topologiques. »

Or ce sont précisément ces collections figurales dont l'informatique «à objets » se laisse de plus en plus fréquemment entraîner à promettre la modélisation efficace, poussée par une demande sociale accrue que concernent directement la fouille de données numériques en ligne, la navigation interactive dans des contenus multimédia ou la recherche d'information au travers de sources multiples. En effet, qu'est-ce qu'écouter de la musique en ligne si ce n'est constituer une collection, quelquefois certes fugace et éphémère, mais toujours figurale en ce sens que sa constitution singulière, sous condition fragile de la continuation, dépend étroitement de la figure temporelle de son déploiement dans la durée?

Notre Faust est donc un collectionneur de fragments d'opéra, auxquels l'informatique va donner les moyens de constituer spatialement et de parcourir ses collections. 


\subsection{Le logiciel ReCollection}

Nous avons travaillé sur ces notions de collections avec plusieurs étudiants, aboutissant à plusieurs prototypes : collections d'objets graphiques 3D et collections de fragments d'opéra. Pour le logiciel ReCollection, il s'agissait de développer un logiciel et des modes d'interface pouvant aussi bien convenir au chanteur sur scène lors de représentations, qu'au spectateur désireux d'approfondir sa connaissance de l'œuvre sur une borne interactive.

A chaque fragment de l'opéra est associé un objet de la collection, auxquels se rapportent une image, un son et un texte. Ces composantes correspondent à des fichiers classiques stockés sur disque (image bitmap, son wave et .txt). Les images de la collection sont des photographies du spectacle. Les sons sont des enregistrements de quelques secondes, comportant la première phrase chantée du fragment choisi. Enfin, les textes reprennent les phrases chantées. Dans le prototype actuel, seuls quelques descripteurs sonores sont utilisés. Ils proviennent d'une analyse réalisée par un patch Max/MSP ad hoc, avec l'objet analyzer $\sim^{10}$.

Pour revenir à notre image de la galerie artistique, les collections sont en général constituées d'un très grand nombre d'œuvres. Cet excès est l'une des caractéristiques nécessaires à la définition d'une collection, comme nous l'avons vu. Lorsque l'on parcourt une réserve, on tombe sur des œuvres une par une, plutôt aléatoirement, et l'on n'a aucune vue d'ensemble sur elles. Dans ce cas, le collectionneur n'a d'autre choix que de passer en revue les éléments un par un, jusqu'à ce qu'il trouve un objet intéressant.

Le logiciel ReCollection organise deux types d'espaces pour ces fragments d'opéra : une ou plusieurs réserve(s) et une ou plusieurs exposition(s). Il implémente deux modes d'actions classificatrices : d'un côté, par similarité calculée, en projetant le résultat de n'importe quelle classification sur les axes de la représentation; de l'autre, par contiguïté « désirée ", permettant à l'utilisateur de placer comme il le souhaite les éléments de sa collection. Il permet de constituer une exposition en important/exportant des objets depuis ou vers la réserve, puis de répartir ces objets dans un espace. Après une exploration de l'état de l'art en visualisation interactive, nous avons choisi un espace à deux dimensions et demi (x, y et échelle), inspiré du logiciel $\mathrm{Pad}++$, la profondeur de la troisième dimension étant difficile à appréhender et manipuler. L'exposition est aussi un lieu d'exploration, autonome ou guidée, qui sera fortement dépendante de la répartition spatiale des objets préalable. Pour explorer l'exposition, l'utilisateur peut déplacer son point de vue horizontalement et verticalement, puis zoomer et 'dézoomer'. Un mode de visite guidée permet de définir/suivre un chemin fixé parmi les objets.

\footnotetext{
${ }^{10} \mathrm{http} / / /$ web.media.mit.edu/ tristan/maxmsp.html
} 


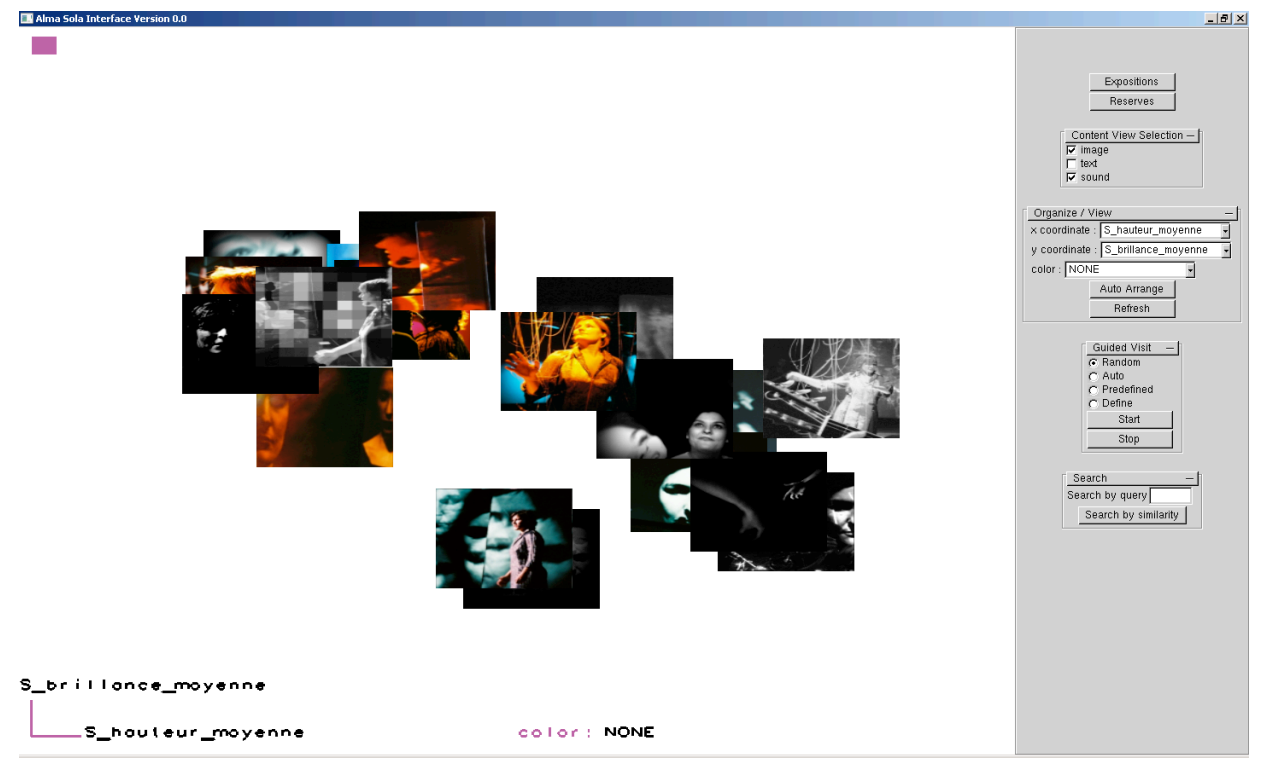

Figure 5. Copie d'écran du logiciel ReCollection. Source : Benjamin Roadley.

La figure 5 montre un ensemble d'icônes associées à des fragments sonores, textuels et visuels d'Alma Sola, agençables en une multitude de collections. La répartition des objets dans l'espace peut être automatique ou manuelle. Le système dispose les objets automatiquement de deux manières différentes :

- La première méthode consiste à calculer les coordonnées spatiales de chaque objet de la collection, ainsi que sa couleur, en fonction des descripteurs sélectionnés par l'utilisateur. Par exemple, celui-ci pourra choisir d'affecter l'énergie sonore à l'axe des abscisses, la luminosité à l'axe des ordonnées, et le temps moyen entre deux attaques sonores à la couleur. L'utilisateur peut aussi affecter un seul des axes, pour aligner les objets sur une droite. Ce mode est celui de la similarité, relevant de l'aspect non-figural des collections ;

- La deuxième méthode pour répartir automatiquement les objets consiste à permettre à l'utilisateur de sélectionner un échantillon de l'exposition. Une analyse est ensuite effectuée sur les valeurs des descripteurs de ces objets, afin d'en tirer les traits caractéristiques, et de répartir tous les objets de la collection selon ces traits. Ce mode est celui de la contiguïté, relevant de l'aspect figural des collections. Ici, une analyse en composante principale calcule une nouvelle base dans l'espace des descripteurs, qui est généralisée à l'ensemble de la collection.

En mode «performance », le fonctionnement de l'application est simplifié, puisque le 
performeur ne dispose que d'une télécommande WiiMote pour manipuler les entités d'opéra et choisir l'un d'entre eux pour continuer l'opéra. Le placement automatique est ici couplé à un modèle d'enchaînement temporel de séquences à base de modèles de Markov cachés (HMM) dans lequel les observables sont les numéros des blocs joués.

\section{Ressaisissement des intentions et mise en scène}

\subsection{Comment traiter les intentions des metteurs en scène?}

Notre problématique relative aux intentions, assez classique en informatique, part d'un besoin exprimé par plusieurs metteurs en scène : peut-on imaginer un environnement informatique permettant de travailler à l'émergence d'éléments de mise en scène et de scénographies ? En complément des approches traditionnelles d'exégèse du texte allant vers son incarnation scénique, les nouvelles technologies laissent imaginer d'autres approches, notamment émergentes : le metteur en scène laisserait jouer ses comédiens en donnant très peu de consignes, voire pas du tout ; un ensemble de dispositifs techniques de captation et d'enregistrement (caméras, microphones, capteurs sur les comédiens, etc.) puis d'analyse et d'extraction d'éléments saillants (traitements informatiques) seraient mis en œuvre et viendraient aider le metteur en scène dans la recherche des formes qu'il voudrait déployer sur scène, puis dans l'implémentation de dispositifs numériques spectaculaires. C'est le sens des ateliers de recherche menés de 2006 à 2008 en collaboration avec la compagnie Le Théâtre du Signe. L'idée était de façonner en même temps outils, méthodes et spectacle.

A l'heure actuelle, la plupart des plateformes de développement liées à la création artistique scénique reposent sur les logiciels graphiques temps réel comme Max/MSP. Il s'agit en général d'y produire des modules interactifs à partir d'une conception de l'œuvre, le plus souvent par prototypage. Mais le développement sur ces plateformes est bien différent des écritures auxquelles on l'associe rapidement, par analogie de domaine : l'écriture musicale et l'écriture de programmes. En effet, l'écriture musicale s'appuie sur les deux paradigmes forts que sont la notation et l'instrument, et de ce fait supporte des systèmes d'intentions, et nous pouvons précisément venir les interpréter dans le rapport à ces deux paradigmes, Par leur rapport à la grammaire et à l'arithmétique, la programmation structurée ou la programmation objets permettent également à celui qui programme comme à celui qui débogue d'imaginer des systèmes d'intentions.

L'informatique musicale temps réel à base de langages graphiques peine à se glisser dans ce type d'écriture et de lecture. Les patchs encodent un ensemble de connaissances et d'intentions qui sont formatées selon un modèle qui les réduit à des flux de signaux. Alors que la programmation objets peine à se saisir des flux, les environnements temps réel à base de flux manipulent en revanche difficilement les structures et les interfaces, et encore moins bien le temps (réduit à un signal). 
C'est pourquoi les patchs codés dans Max/MSP ou PureData sont difficiles à relire et maintenir. Le fouillis qu'autorise la simplicité du mode de développement (par connexion, équivalent d'un fil électrique) n'arrange pas les choses, et l'on comprend bien pourquoi leurs concepteurs peinent à les relire dès qu'ils en ont terminé l'agencement.

\subsection{Etat de l'art et propositions contemporaines}

Un certain nombre de développeurs et d'artistes, agacés par les défauts des plateformes graphiques temps réel, ont commencé à produire et diffuser des environnements dans lesquels l'intention du concepteur ne serait pas noyée dans les primitives du traitement du signal. Plusieurs axes de travail se sont dégagés :

- L'écriture temporelle de l'interaction : si l'on considère l'informatique musicale avant tout sous l'angle du déclenchement, on imagine la nécessité d'un codage temporel d'une partition interactive, autorisant une certaine souplesse dans la mise en œuvre par un performeur humain. Le modèle sous- jacent est la partition interactive ou " partition virtuelle » au sens de Philippe Manoury [Manoury \& Battier 1987] ${ }^{11}$, que l'on aurait simplifiée dans ses modalités expressives au profit d'une modélisation temporelle forte. Les travaux d'Antoine Allombert, Myriam Desainte-Catherine et Gérard Assayag sur le système iScore [Desainte-Catherine \& al. 2005] donnent une bonne idée de l'état de l'art du domaine ;

- L'écriture spatiale de l'interaction : un certain nombre de chercheurs s'orientent vers une "spatialisation » de la conception et du rendu d'œuvres interactives. Nous avions travaillé il y a quelques années sur le sujet et abouti au système ALMA de composition de "scènes musicales 》 spatialisées. En général, ces approches proposent des espaces tri-dimensionnels dans lesquels sont intégrés des contenus visuels et sonores. Le modèle sous-jacent est le paysage sonore, que le visiteur vient parcourir et altérer en situation (déclenchements et transformations). Les travaux d'Antonio de Sousa Dias sur l'application InstallaSon et les différents usages de la plateforme VirChor développée par Christian Jacquemin [Jacquemin 2004] sont représentatifs de cette orientation de recherche ;

- La recherche sur des «grammaires » de l'interaction : certains chercheurs se sont intéressés à la modélisation de l'interaction en relation avec des langages de programmation. Antonio Camurri [Camurri \& al. 1991] et son équipe ont développé un système appelé Harp, comportant deux niveaux de manipulation de connaissances musicales : un environnement orienté objets pour la gestion du son (échantillons, fragments de codes, algorithmes) et un environnement symbolique de gestion des règles compositionnelles et des partitions de plus haut niveau. Yann Orlarey a quant à lui développé un langage, appelé Faust, de description générique

\footnotetext{
${ }^{11}$ Philippe Manoury lui-même réfléchit à « une écriture nouvelle pour la musique de synthèse qui permettrait également d'être un outil puissant pour la composition ». Cf. [Manoury 2007].
} 
de traitements temps réel [Orlarey \& al. 2002].

Notre recherche se polarise avant tout sur le processus de conception de l'œuvre interactive, tentant de s'y glisser pour permettre d'en exhiber, dans le dialogue homme-machine, des intentions. Ce qui ne veut pas dire que nous ne serons pas amenés à utiliser tel ou tel composant développé et diffusé par des chercheurs abordant l'une des trois directions que nous venons de citer. Enfin, nous avons travaillé sur un environnement destiné à la scénographie et au théâtre plutôt qu'à la composition musicale - cette dernière n'est évidemment pas exclue, et nous l'avons expérimenté dans le cadre du projet Les petites absences.

\subsection{Vers un assistant virtuel de metteur en scène et performeur}

Nous avons développé un projet d'assistant virtuel de metteur en scène et performeur, qui aborde la problématique selon deux axes de travail :

- La spécification de l'interaction homme-machine ;

- La conception scénographique comme façonnage émergent de formes.

Ces deux volets nous renvoient à deux paradigmes de l'interaction homme-machine temps réel en contexte scénique, au sens large :

- Le paradigme de la partition ${ }^{12}$ : il y a dans tout texte écrit, qu'il soit une partition ou un texte de théâtre, l'espace qu'ouvre l'interprétation, cet écart entre le réalisé et l'attendu ; c'est dans ce sens que Philippe Manoury définit les «partitions virtuelles » [Manoury \& Battier 1987] : " une partition virtuelle est une organisation musicale dans laquelle on connaît la nature des paramètres qui vont être traités, mais pas toujours la valeur exacte qu'ils vont recevoir le moment voulu. Une partie de ces paramètres sera fixe et ne changera pas d'une interprétation à l'autre, une autre sera influencée par la captation d'événements extérieurs (un micro, par exemple) qui sera analysée et traitée suivant certaines règles de composition. » Dans cette perspective, l'accent est mis sur une résolution analytique de plus en plus fine dans la captation de tel ou tel son au sein d'un ensemble; et désormais dans le suivi de tel ou tel geste du musicien, intégrant une dimension visuelle et corporelle indispensable: "Le fait que des musiciens puissent jouer à l'intérieur d'un temps commun est un phénomène grandement visuel. C'est par un petit signe de la tête ou du bras qu'ils commencent ensemble. La continuité du geste $d u$ chef d'orchestre envoie sans interruptions, des signaux prédictifs qui orientent les actions musicales dans la direction d'un futur proche. On devine où va " tomber » le deuxième, le troisième puis le quatrième

\footnotetext{
${ }^{12}$ Lorsque l'on examine la partition d'Anthèmes 2 de Pierre Boulez pour violon et électronique temps réel, on est frappé par l'inscription des actions des différents modules électroniques : en effet, Andrew Gerzso, assistant du compositeur, a dans la mesure du possible réécrit selon une notation musicale les transformations opérées par les différents modules informatiques.
} 
temps. » ;

- Le paradigme de l'instrument, plutôt que celui de la partition. Envisageons ici l'instrument sous son double visage phénoménologique : l'apprentissage et la surprise. Les dispositifs interactifs peuvent ouvrir la porte aux moments où les similarités, et donc une forme de sens, se façonnent dans le dialogue homme- machine à partir des situations qui se présentent. Comme l'instrument de musique que nous ne cessons de travailler et qui ne cesse de nous surprendre, ils donneront lieu à l'émergence d'inattendus. Est émise ici l'idée - qui peut surprendre de prime abord - d'une informatique temps réel comme condition de possibilité de la singularité.

$\mathrm{Au}$ cours de nos travaux, nous avons cherché un certain équilibre, en distribuant les analyses, les fonctionnalités et les développements, autour de ces deux paradigmes.

\section{Assistant virtuel de mise en scène}

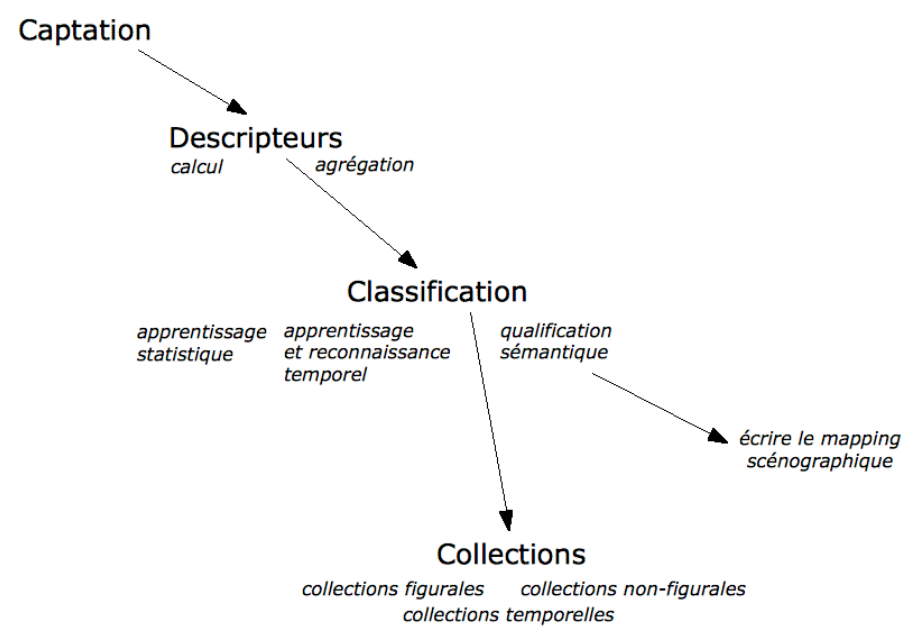

Figure 6. Schéma de principe de l'assistant virtuel. Source : Alain Bonardi.

\subsection{Conception d'ensemble de l'assistant virtuel}

L'assistant virtuel comporte deux parties fonctionnelles : d'une part, une partie « façonnage » pour le repérage et la compréhension d'états et de séquences intéressants aux yeux du metteur en scène; d'autre part, une partie « régie » permettant d'esquisser une scénographie numérique. La figure 6 en montre le schéma de principe.

L'assistant est conçu dans une approche bottom-up, des signaux captés en observant les performers en passant par des étapes de classification, de description sémantique, jusqu'aux collections de fragments scéniques tels que décrits dans la partie précédente de ce texte. 


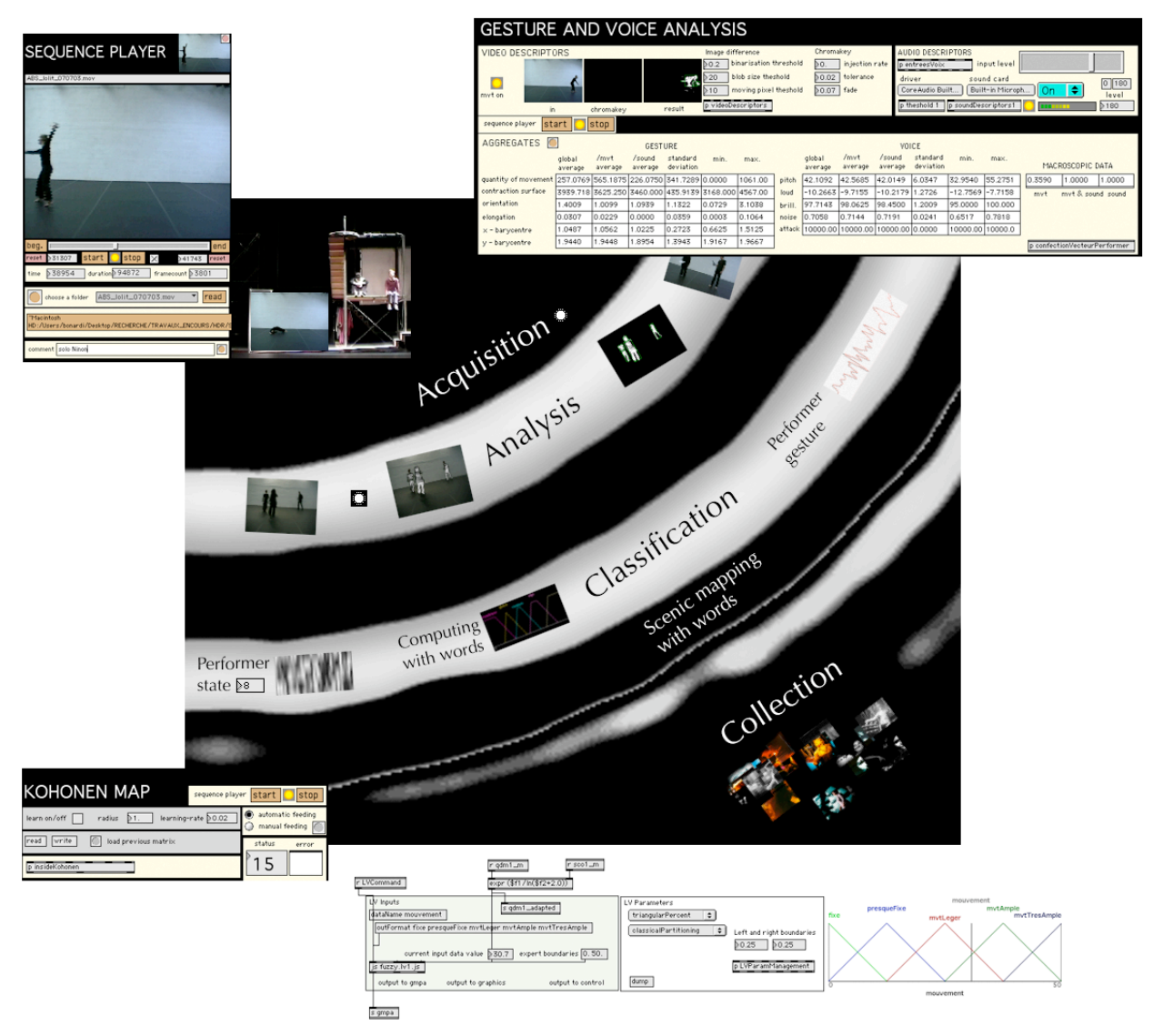

Figure 7. Implémentation Max/MSP de l'assistant virtuel. Source : Alain Bonardi.

La figure 7 montre en copie d'écran le patch Max/MSP tel qu'il a été implémenté pour le projet Les Petites Absences ${ }^{13}$ : au centre, le module principal, calqué sur le schéma de principe de la figure 6 , et autour, en « développé » les sous-modules dédiés aux différentes fonctions.

\section{5. «L'assistant-façonnage » au coeur du projet Les petites absences}

Détaillons maintenant le premier volet de notre assistant virtuel, destiné à l'élaboration extensive, par le dialogue homme-machine, "d'atomes » de scénographie et de mise en scène. Nous le nommons « assistant-façonnage »; il s'agit à partir d'un grand nombre de données (descripteurs vidéo et audio du performeur), de constituer des abstractions de manière automatique, mais également de manière interactive. Les fonctionnalités principales concernant les matériaux dramaturgiques sont : l'acquisition, l'annotation, la classification, la sélection, la reconnaissance. Le système actuel comporte les composants

\footnotetext{
${ }^{13}$ Les petites absences est une création théâtrale, musicale et technologique, dont la première a été donnée le 16 décembre 2008 à la Comédie de Caen. Sa thématique est l'absence, comme présence qui devient obsédante. Le projet fait appel à un dispositif numérique appelé LAB100, qui gère des « traces » des absents et les confronte aux présents. Texte : Sylvie Robe ; mise en scène : Marco Bataille-Testu ; création images : Nicolas Girault; chorégraphie : Lolita Espin Anadon ; création musicale : Alain Bonardi.
} 
suivants, tous développés avec Max/MSP :

- Un lecteur multimédia (vidéo et audio) permettant de repérer des passages-clé, de les annoter, et d'en prendre une photographie annotée (en haut à gauche sur la figure 7);

- Un module d'acquisition vidéo et audio, calculant un ensemble de descripteurs. L'analyse de la vidéo est effectuée grâce aux objets de la librairie Computer Vision (cv.jit) pour Max/MSP. Un double traitement, d'une part différentiel, d'autre part en suivi de couleur est effectué en entrée, avec la possibilité de doser la part de chacun. Puis, un certain nombre de descripteurs instantanés sont calculés à partir des rectangles enveloppant les performeurs : quantité de mouvement, surface de contraction, orientation, élongation, centre de gravité. Pour l'audio, à partir de l'objet analyzer de Tristan Jehan, nous obtenons un ensemble de descripteurs audio : volume sonore, brillance, degré de bruit, durée entre deux attaques (en haut à droite sur la figure 7);

- Un module de calcul d'agrégateurs à partir des descripteurs ci-dessus. Ce sont des moyennes, écarts-types, minima et maxima, obtenus à partir des librairies FTM et $M n M$. Ils sont calculés selon une périodicité variable, modulable par l'utilisateur (fixe, selon des seuils par rapport au volume sonore, ou globalement pour une séquence) ; - Un module d'apprentissage non supervisé, correspondant à une carte de Kohonen [Kohonen 1982], permettant la classification des vecteurs d'agrégateurs en classes d'état du performeur. Ce module utilise l'objet jit.robosom pour Max/MSP. En l'état actuel du système, 16 classes d'états du performeur ont été distinguées (en bas à gauche, sur la figure 7);

- Un module de suivi et de réalignement temporel prenant en compte l'évolution de l'état du performeur dans le temps. Il utilise les patchs du suiveur de geste mnm.follower développé par Frédéric Bevilacqua [Bevilacqua \& al. 2007], fondé sur des modèles de Markov cachés (HMM) permettant un apprentissage rapide (en une seule passe) de « gestes dramaturgiques ».

- Un module de façonnage interactif de listes de séquences, permettant au concepteur de rassembler plusieurs séquences qui lui semblent «aller ensemble » et d'obtenir les descripteurs " principaux » qui caractérisent cette liste, par une analyse en composante principale. Ce module utilise le moteur du logiciel ReCollection, présenté au paragraphe précédent.

Dans l'état actuel de cette partie du logiciel, il est utilisé par le metteur en scène Marco Bataille-Testu pour trois types d'annotations, correspondant à trois manières de se forger des idées de mise en scène :

- Exhiber d'un point de vue dramaturgique et nommer les classes d'états du performeur définies par la carte de Kohonen;

- Repérer et mémoriser des moments dramaturgiques intéressants qui seront « appris » par le module de suivi et réalignement temporel, et par la suite reconnus ; 
- Constituer des familles de séquences de performances, selon un fonctionnement en collection, à savoir passant des propositions de contigüité (éprouvée) aux propositions de similarité (calculée).

\section{6. «L'assistant-régie » et la « Fuzzy Lib»}

L'autre versant de l'assistant virtuel est la partie « assistant-régie » qui spécifie l'interaction homme-machine à mettre en œuvre, à savoir les relations entre entrées et sorties du système informatique développé. Nous sommes là dans un univers de fonctions, de calculabilité de cette interaction sur une machine de Turing.

Mais comment prolonger la fiction dans le monde des fonctions ? Il s'agirait non pas de supprimer les fonctions, car toute machine de Turing en a besoin, mais bien de les alléger, et en tout cas de diminuer l'importance des « grilles » de possibles qui structurent par défaut toute réalisation interactive.

Nous nous sommes intéressés à la logique floue pour un ensemble de raisons :

- L'appariement entre entrées et sorties pourrait s'écrire sous forme de règles floues plutôt que se coder sous forme de fonctions ;

- L'abandon des fonctions au profit d'une sémantique utilisateur (fuzzification et règles floues) favorise selon nous une plus grande autonomie des concepteurs non aguerris au développement Max/MSP et souvent bloqués par l'aspect fonctionnel implicite dans ce type d'environnement ;

- La logique floue associe de manière intuitive perception et action dans le monde numérique, où les variations dans les espaces de valeurs de certains paramètres correspondent rarement au retour sensoriel que l'on en a. Un partitionnement astucieux sur des variables linguistiques permet ainsi de nommer des espaces de valeurs en phase avec la perception ;

- Enfin, la logique floue nous apporte une vision intéressante de la classification, qui pourrait contribuer à l'unification des deux parties de l'assistant : « façonnage » et « régie ». En effet, nous pouvons imaginer que des règles floues induites à partir de sélections du metteur en scène ou concepteur, constituent la base de celles qui seraient utilisées pour la régie du spectacle.

Nous avons donc développé une bibliothèque temps réel de logique floue intitulée FuzzyLib pour Max/MSP. Cette implémentation [Bonardi \& Truck 2009], téléchargeable à l'adresse http://imtr.ircam.fr propose la plupart des implications floues, t-normes et t- conormes de la littérature [Bouchon-Meunier 1995].

Dans le projet Les petites absences, une bonne partie de la régie " temps réel » est implémentée en utilisant des règles de logique floue, qui manipulent des variables 
linguistiques correspondant en sortie à des paramètres de modules interactifs : le bas de la figure 7 montre la modélisation de la quantité de mouvement (nombre de pixels ayant varié entre deux frames) du performeur selon 5 sous-ensembles flous (« fixe », "presqueFixe ", « mouvementLéger », " mouvementAmple », « mouvementTrèsAmple »). Le dispositif a été adopté par le metteur en scène Marco Bataille-Testu pour deux raisons principales : la facilité à créer des correspondances entre entrées et sorties en posant des règles floues, alors que la manipulation directe de Max/MSP lui pose des problèmes ; l'ouverture au vocabulaire du projet théâtral mené, qui est directement utilisé dans la modélisation des phénomènes (en l'occurrence, les 5 sous-ensembles flous de la quantité de mouvement ont été « baptisés » par le metteur en scène).

\section{Conclusion}

\subsection{La médiation de l'activité scénique vue selon deux problématiques informatiques}

Nous avons montré comment deux questions centrales de l'informatique pouvaient nourrir la recherche sur les médiations technologiques pour la création scénique. Trois spectacles ont été présentés, et ce croisement entre problématiques informatiques versus conceptions de mise en scène a permis d'esquisser des thématiques plus larges :

- L'étude de cas autour de La traversée de la nuit montrait les premiers pas d'une démarche vers la constitution de la scène comme Environnement Virtuel Informé ;

- L'étude de cas autour d'Alma Sola montrait toute l'importance du paradigme cognitif des collections ;

- L'étude autour des Petites absences suggérait l'importance de la ressaisie d'intentions au cœur des processus de conception d'un spectacle interactif, avec notamment l'utilisation de la logique floue pour permettre l'insertion du vocabulaire des utilisateurs et sa manipulation.

\subsection{Trois combinaisons d'utilisateurs et de lieux de créativité à explorer}

Jusqu'ici, nous avons peu évoqué les utilisateurs de nos systèmes. Nous pouvons en distinguer trois catégories : les artistes concepteurs, les artistes performeurs en situation scénique, et le public. Nous avons surtout insisté sur des médiations technologiques à l'attention du premier groupe, mais les deux autres sont évidemment tout aussi dignes d'intérêt.

Chacun groupe est associé à un ou plusieurs espaces dans lesquels les utilisateurs opèrent des manipulations créatives de contenus. Une compréhension située de ces modes d'interaction nous semble être une piste de travail tout à fait intéressante :

- Pendant les répétitions, les artistes concepteurs circulent entre deux lieux : ils ne 
cessent de passer de la table à la scène. Le carnet de notes présente l'avantage de pouvoir être transporté avec soi dans ce mouvement de va-et- vient et de ne pas interrompre le processus d'élaboration, tout particulièrement le dialogue avec les performeurs. En revanche, l'ordinateur, placé sur la table de travail au niveau des fauteuils de salle, immobilise le concepteur, et requiert toute son attention, lui faisant perdre le fil de l'échange, du dialogue. Or la scène est souvent, dans ces productions, équipée en moyens de projection visuelle et sonore, qui sont essentiellement utilisés pour la régie et très rarement comme supports de conception d'un spectacle. On continue à placer sur scène des traits à la craie, ou encore des morceaux de scotch coloré pour se repérer. Ne peut-on pas imaginer d'étendre l'introduction des nouvelles technologies dans la conception de spectacles à une écriture en situation, utilisant pleinement tous les moyens disponibles ? A quelles fonctionnalités et quelles interfaces pouvons-nous penser? Nous imaginons alors un Environnement Virtuel Informé pour concepteur, qui intègre un certain nombre de technologies dans son double espace de travail : à la table, et sur scène ;

- Pour les artistes performeurs qui jouent sur scène, l'espace auquel nous pensons immédiatement est évidemment le plateau. Mais l'évolution sociale qui accompagne l'évolution technologique montre une individuation marquée des pratiques, et un certain nombre d'activités autrefois collectives sont désormais réalisées à domicile. La résidence du comédien, du musicien ou du danseur n'est pas seulement le lieu de sa pratique quotidienne, de son apprentissage privé. Elle pourrait devenir un espace de training assisté par ordinateur, généralisant la fonction du miroir, bien connue des danseurs. A savoir provoquer une altérité par un artefact : le miroir nous donne une image impossible de nous-mêmes, qui nous interroge et nous fait réagir. Nous trouvons particulièrement riche cette thématique, en la transposant dans le monde numérique : quels seraient les doubles, les avatars au sens large qui pourraient nous provoquer, et quelles situations d'interaction pourrions-nous imaginer avec eux ? Nous aurions à penser un Environnement Virtuel Informé bipolaire pour les performeurs, entre le plateau et le lieu d'apprentissage ;

- Enfin, pour les spectateurs, la situation est encore plus complexe, puisque les possibilités de " lieux » de spectacle se multiplient, allant de la salle de concert jusqu'au baladeur, en passant par le domicile, les espaces collectifs non exclusivement scéniques comme les musées par exemple, voire les façades d'immeubles. Le spectacle numérique est partout, et ses interfaces désormais innombrables : le spectateur " actif» procède à une forme de recréation de contenus artistiques par la médiation de machines. Nous nous intéressons à deux « lieux » particuliers : les salles de spectacles ouvertes à cette médiatisation de la recréation, et les dispositifs portables (téléphone, baladeur, etc.) la permettant. Chaque situation est en soi un projet de recherche, et requiert la mise au point d'imaginatifs Environnements Virtuels Informés. 


\section{Références bibliographiques}

[Bablet 1995] BABLET, Denis (sous la direction de), L'œuvre d'art totale, Paris, CNRSEditions, 1995.

[Banu 2005] BANU, Georges (éditeur), Les Répétitions - de Stanislavski à aujourd'hui, Le Méjan : Actes Sud, 2005, 440 pages.

[Benjamin 1989] BENJAMIN, Walter, Paris, capitale du XIX siècle - le livre des passages, Paris : Le Cerf, 1989, 967 pages.

[Baudrillard 1988] BAUDRILLARD, Jean, Le système des objets, Paris : Gallimard, 1988, 275 pages.

[Bevilacqua 2007] BEVILACQUA, Frédéric, GUEDY, Fabrice, SCHNELL, Norbert, FLETY, Emmanuel, LEROY, Nicolas, Wireless sensor interface and gesture-follower for music pedagogy, Proceedings of the New Interfaces for Music Expression International Conference (NIME07), pages 124-129, New York (USA), 7-9 juin 2007.

[Bonardi 2008] BONARDI, Alain, Représentations opérationnelles, Sampzon : Editions Delatour France, 2008, 302 pages.

[Bonardi \& Truck 2009] BONARDI, Alain, TRUCK, Isis, « Designing a library for computing [performances] with words», Proceedings of the Fourth International Conference on Intelligent Systems and Knowledge Engineering, Hasselt (Belgique), 2009.

[Boniol \& al. 2003] BONIOL, Frédéric, BEL, Gérard, ERMONT, Jérôme, "Trois approches pour la modélisation et la vérification de systèmes embarqués », Technique et Science Informatiques, Hermes, Vol. 22, N. 5, p. 539-569, 2003.

[Bouchez \& Leleu-Merviel 2001] BOUCHEZ, Pascal, LELEU-MERVIEL, Sylvie, La simulation de représentation - Dispositif de co-production sémantique pour l'écriture de traces audiovisuelles respectueuses du fait théâtral, in Hypertextes-Hypermédias H2PTM'01, actes de la conférence H2PTM'01, octobre 2001, Valenciennes, Paris : Hermes Science Publications, 2001, 416 pages.

[Bouchon-Meunier 1995] BOUCHON-MEUNIER, Bernadette, La Logique floue et ses applications, Paris : Addison-Wesley, 1995.

[Bougnoux 2006] BOUGNOUX, Daniel, La crise de la représentation, Paris : La Découverte, 2006, 183 pages.

[Camurri \& al. 1991] CAMURRI, Antonio, CANEPA, Corrado, FRIXIONE, Marcello, ZACCARIA, Renato, HARP: A System for Intelligent Composer's Assistance, IEEE Computer 24(7): 64-67 (1991). 
[Cavazza \& al. 2002] CA V AZZA, Marc, CHARLES, Fred, MEAD, Steven J., CharacterBased Interactive Storytelling, IEEE Intelligent Systems 17(4): 17-24 (2002).

[Desainte-Catherine \& al. 2005] DESAINTE-CATHERINE, Myriam, ALLOMBERT, Antoine, Interactive scores: a model for specifying temporal relations between interactive and static events, Journal of New Music Research, Vol. 34, N 4, 2005.

[Dixon 2007] DIXON, Steve, Digital Performance : A History Of New Media In Theatre, Dance, Performance Art And Installation, Mit Press, 2007.

[Ferber 1995] FERBER, Jacques, Les systèmes multi-agents, Paris : InterEditions, 1995, 493 pages.

[Jacquemin 2004] JACQUEMIN, Christian, Architecture and Experiments in Networked 3D Audio/Graphic Rendering with Virtual Choreographer, actes de la Conférence Internationale Sound \& Music Computing (SMC'04), Paris, 20-22 octobre 2004.

[Kohonen 1982] KOHONEN, Teuvo, Self-organized formation of topologically correct feature maps, Biological Cybernetics 43, 59-69.

[Manoury 2007] MANOURY, Philippe, Considérations [toujours actuelles] sur l'état de la musique en temps réel, Revue l'Etincelle, Prospectives, Paris : Ircam - Centre Georges Pompidou, 2007.

[Manoury \& Battier 1987] MANOURY, Philippe, BATTIER, Marc, Les partitions virtuelles, Documentation Ircam, Paris, 1987.

[Orlarey \& al. 2002] ORLAREY, Yann, FOBER, Dominique, LETZ, Stéphane, « An algebraic approach to block diagram constructions », Actes des Journées d'Informatique Musicale 2002 (JIM 2002), Marseille : GMEM, 2002.

[Pachet 2004] PACHET, François, Les nouveaux enjeux de la réification. In L'Objet, 10(4), 2004.

[Perrot 1994] PERROT, Jean-François, Des objets aux connaissances, Journée Méthodes objets et Intelligence Artificielle : Frontières, Ponts et Synergies, Paris RIA, 1994.

[Piaget \& Inhelder 1966] PIAGET, Jean, INHELDER, Barbet, La psychologie de l'enfant, Paris : Presses Universitaires de France, 1966.

[Peyret 1998] PEYRET Jean-François, « Texte, scène et vidéo », Les écrans sur la scène, Béatrice Picon-Vallin éd., Paris : L'âge d'homme, 1998, p. 279-294.

[Picon-Vallin 1998] PICON-VALLIN, Béatrice, «Hybridation spatiale, registres de présence », Les écrans sur la scène, Béatrice Picon-Vallin éd., Paris : L'âge d'homme, 
1998, p. 9-35.

[Pistone 1994] PISTONE, Danièle, « Chopin et le modernisme musical français. Un aspect de la réception de l'œuvre chopinienne dans la France du XX $\mathrm{X}^{\mathrm{e}}$ siècle », (Colloque international Chopin de Varsovie, 1986), Chopin Studies (Warszawa), n 4, 1994, p. 116120.

[Polieri 1963] POLIERI, Jacques, Scénographie : théâtre, cinéma, télévision, Réédition revue et augmentrée de Scénographie nouvelle (1963), 1990, Paris : Editions Jean- Michel Place.

[Pomian 1987] POMIAN, Krysztof, Collectionneurs, amateurs et curieux, Paris : Gallimard, 1987, 367 pages.

[Reaney 2001] REANEY, Mark, Virtual characters in theatre production : actors and avatars, actes de la conférence Laval Virtual 2001, Laval, 2001.

[Rousseaux 2006a] ROUSSEAUX, Francis, La collection, un lieu privilégié pour penser ensemble singularité et synthèse, revue en ligne EspacesTemps, 2006, accessible à l'URL : http://www.espacestemps.net/document1836.html.

[Rousseaux 2006b] ROUSSEAUX, Francis, Singularités à l'œuvre, Sampzon : Delatour, 2006, 2 volumes, 361 et 339 pages.

[Rousseaux \& Bonardi 2005] ROUSSEAUX, Francis, BONARDI, Alain, Similarité en intension vs en extension : à la croisée de l'informatique et du théâtre, in Revue d'intelligence artificielle (RIA 05), Volume 19, $N^{\circ} 1-2 / 2005$, Paris, Hermès-Lavoisier, pages 281-288.

[Schaeffer 1966] SCHAEFFER, Pierre, Traité des objets musicaux, Paris : Seuil, 1966.

[Turing 1995] TURING, Alan, Computing Machinery and Intelligence, Mind LIX, $\mathrm{n}^{\circ} 236$, 1950 ; réédité dans les Collected Works of A-M. Turing, vol 3. "Mechanical Intelligence"; traduction française dans A-M. Turing, J.-Y. Girard, La machine de Turing, Paris : Seuil, 1995.

[Wajcman 1999] WAJCMAN, Gérard, Collection, Paris : Nous, 1999, 110 pages.

[Zadeh 1997] ZADEH, Lofti A., Toward a theory of fuzzy information granulation and its centrality in human reasoning and fuzzy logic, Fuzzy Sets and Systems 90 (1997) 111-127.

[Zeppenfeld-Rosaz \& Le Brian-Prada 1998] ZEPPENFELD-ROSAZ, Christine, LE BRIAN-PRADA, Nicole, « De l'installation à la performance : la tradition au cœur de la technologie », Les écrans sur la scène, Béatrice Picon-Vallin éd., Paris : L'âge d'homme, 1998, p. 257-278. 
Alain Bonardi est maître de conférences habilité à diriger des recherches à l'Université Paris 8 , en délégation à l'IRCAM. Ses thématiques de recherche concernent l'application de l'informatique et de l'intelligence artificielle au spectacle vivant.

Francis Rousseaux est professeur à l'Université de Reims Champagne-Ardenne et collabore régulièrement avec l'Ircam. Ses travaux portent sur les possibles extensions des Collections figurales de Piaget à des outils d'aide à la navigation socio-sémantique dans le Web 2.0. 